\title{
Polymer-assisted synthesis of ethyl 2-amino-4,6-diarylpyrimidine-5-carboxylates
}

\author{
Jean Jacques Vanden Eynde, ${ }^{* a, b}$ Nadège Labuche, ${ }^{\text {b, c }}$ Yves Van Haverbeke, ${ }^{b}$ and Lutz \\ Tietze $^{c}$ \\ ${ }^{a}$ Xavier University of Louisiana, College of Pharmacy, \\ Division of Basic Pharmaceutical Sciences, 1 Drexel drive, New Orleans LA 70125, USA \\ ${ }^{b}$ University of Mons-Hainaut, Organic Chemistry Department, 20 place du Parc, \\ B-7000 Mons, Belgium \\ ${ }^{c}$ Institut für Organische Chemie, Georg-August-Universität Göttingen, Tammannstrasse 2, \\ D-37077 Göttingen, Germany \\ E-mail:jjvanden@xula.edu
}

(received 11 June 03; accepted 09 Sept 03; published on the web 10 Oct 03)

\begin{abstract}
Nine novel ethyl 2-amino-4,6-diarylpyrimidine-5-carboxylates have been synthesized in modest to good yields by a five-step procedure that involves building of the heterocyclic moiety on a solid support derived from Merrifield's resin and final displacement with an amine.
\end{abstract}

Keywords: Biginelli compounds, Merrifield's resin, oxidation, pyrimidine, solid-phase synthesis

\section{Introduction}

Recently we focused our attention on derivatives of pyrimidine-5-carboxylic acid, ${ }^{1}$ some of them exhibiting antihypertensive, ${ }^{2}$ antiviral, ${ }^{3}$ antianoxis, ${ }^{4}$ and antilipid peroxidation ${ }^{4}$ activities. In addition, this cyclic system can be found in the crambine alkaloids. ${ }^{5}$ Such subtances (4, Scheme 1) are readily available by the Atwal modification ${ }^{6}$ of the Biginelli ${ }^{7}$ multi-component reaction, i.e. interaction between an amidine-like compound $\mathbf{1}$ and a 2-alkylidene-3-ketoester $\mathbf{2}$, followed by an oxidation of the intermediate 1,4 dihydropyrimidine 3 .

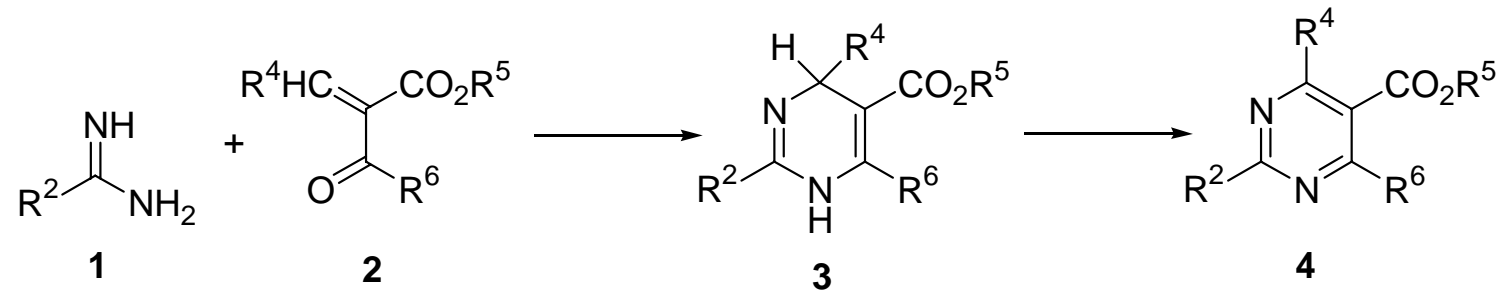

Scheme 1. Preparation of derivatives of pyrimidine-5-carboxylic acid. 
Among the pyrimidines 4, products bearing an amino group in position $2\left(R^{2}=-N R R\right.$ ') are poorly described in the literature probably because of the lack of straightforward synthetic methods. Indeed, the amino moiety can be introduced during the ring formation process with an appropriate guanidine $\left(1, \mathrm{R}^{2}=-\mathrm{NRR}\right.$ ') but the approach is of restricted interest because of the limited availability of substituted guanidines. The amino moiety can also be introduced by displacement of a good leaving group with an amine after construction of the heterocycle $\mathbf{3}$ or 4 . However, to the best of our knowledge, that strategy has been seldom exploited to access the title compounds. In this paper we wish to report on the preparation of the target substances from alkyl 2-(alkylthio)pyrimidine-5-carboxylates bound to a solid support through the thio group, reasoning that an aminolysis reaction could effect the cleavage from the polymer while inducing the expected functionalities in position 2 .

\section{Results and Discussion}

Our strategy (Scheme 2) started by the modification of the Merrifield's resin into 5 by reaction with thiourea. Condensation of 5 with a pre-formed ${ }^{8}$ ethyl 3-aryl-2-benzoylpropenoate (6) gave the polymer-supported 1,4-dihydropyrimidine 7, which was oxidized by treatment with ceric ammonium nitrate ${ }^{1 \mathrm{a}, 9}$ to afford $\mathbf{8}$. The formation of the polymer-bound compounds was followed on line by infrared spectroscopy. Disappearance ${ }^{10}$ of the $\mathrm{C}-\mathrm{Cl}$ band at $1264 \mathrm{~cm}^{-1}$ and appearance of a broad absorption ${ }^{11}$ around $3435 \mathrm{~cm}^{-1}$ are indicative for the fixation of the thiourea. The presence of a carbonyl peak at $1680 \mathrm{~cm}^{-1}$ suggests the incorporation of the ester moiety (in 7) and that band is shifted to $1720-1730 \mathrm{~cm}^{-1}$ in the corresponding aromatic derivative (8). Inspection of the infrared spectra revealed that construction of the 1,4-dihydropyrimidine system on the polymer was the crucial step that determined the overall yield. We also observed that reacting together the solid 5, an aldehyde, and a $\beta$-ketoester (the Hideg modification ${ }^{12}$ of the Biginelli synthesis) was a less attractive method, as formation of by-products in solution occurred more rapidly than the interaction with the solid reagent.

Disappointingly, we found that displacement of the alkylthio group in $\mathbf{8}$, by an amine, was a slow process. Therefore, we turned towards the concept described by Obrecht ${ }^{11,13}$ who suggested transforming the alkylthio group into the more labile sulfone by treatment with metachloroperoxybenzoic acid. It was not possible to convert $\mathbf{7}$ into $\mathbf{9}$ directly, thus justifying the use of two oxidants. Finally, the cleavage from the resin was readily accomplished with various aliphatic or aromatic primary and secondary amines in boiling ethanol. Overall yields (not optimized), based on the chlorine content of the Merrifield's resin, ranged from 35 to $75 \%$ as indicated in the Table. 
Table. Nature of the substituents in compounds $\mathbf{1 0}-\mathbf{1 8}$ and overall yields

\begin{tabular}{ccccc}
\hline Cpd & $\mathrm{Ar}$ & $\mathrm{R}$ & $\mathrm{R}^{\prime}$ & Overall Yield (\%) * \\
\hline $\mathbf{1 0}$ & $\mathrm{Ph}$ & $\mathrm{Me}$ & $\mathrm{Me}$ & 75 \\
$\mathbf{1 1}$ & $\mathrm{Ph}$ & $\mathrm{Ph}$ & $\mathrm{H}$ & 35 \\
$\mathbf{1 2}$ & $\mathrm{Ph}$ & $4-\mathrm{Cl}-\mathrm{Ph}$ & $\mathrm{H}$ & 55 \\
$\mathbf{1 3}$ & $\mathrm{Ph}$ & 4-(Ome)-Ph & $\mathrm{H}$ & 65 \\
$\mathbf{1 4}$ & $\mathrm{Ph}$ & $-\left(\mathrm{CH}_{2}\right)_{4}-$ & 50 \\
$\mathbf{1 5}$ & $\mathrm{Ph}$ & $-\left(\mathrm{CH}_{2}\right)_{2}-\mathrm{O}-\left(\mathrm{CH}_{2}\right)_{2}-$ & 40 \\
$\mathbf{1 6}$ & $\mathrm{Ph}$ & $\mathrm{But}$ & $\mathrm{H}$ & 70 \\
$\mathbf{1 7}$ & 4-(NO $\left.\mathrm{NO}_{2}\right)-\mathrm{Ph}$ & $\mathrm{Me}$ & $\mathrm{Me}$ & 65 \\
$\mathbf{1 8}$ & 4-(Ome)-Ph & $\mathrm{Me}$ & $\mathrm{Me}$ & 70 \\
\hline
\end{tabular}

*: not optimized.<smiles>CCOC(=O)CC(=O)c1ccccc1</smiles><smiles>CCOC(=O)/C(=C\Br)C(=O)c1ccccc1</smiles><smiles>OCCl</smiles>

Merrifield's resin<smiles>NC(=S)S</smiles>

ii

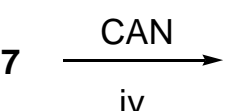<smiles>CCCc1cnc(SCO)nc1O</smiles>

8

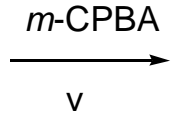<smiles>[R]NPCC</smiles><smiles>[R]N([R])c1nc([Al])c(C(=O)OCC)c(C(=O)OCC)n1</smiles>

i: $\mathrm{C}_{6} \mathrm{H}_{6}$, Dean-Stark

ii: EtOH, reflux, $16 \mathrm{~h}$

iii: DMF, $\mathrm{NaHCO}_{3}, 70^{\circ} \mathrm{C}, 16 \mathrm{~h}$

iv: $\mathrm{CH}_{2} \mathrm{Cl}_{2}, \mathrm{H}_{2} \mathrm{O}, \mathrm{rt}, 24 \mathrm{~h}$

v: $\mathrm{CH}_{2} \mathrm{Cl}_{2}, \mathrm{rt}, 24 \mathrm{~h}$

vi: $\mathrm{EtOH}$, reflux, $16 \mathrm{~h}$

Scheme 2. Procedure used to prepare compounds 10 - 18 . 


\section{Conclusions}

In this paper we present our preliminary results on a simple strategy to access the title compounds. From these results, it can be anticipated that the sequence might be suitable for a combinatorial approach. Indeed substituents $\mathrm{R}^{4}$ and $\mathrm{R}^{6}$ could be independently modified, as well as the nature of the ester group $\left(\mathrm{R}^{5}\right)$. However, to reach that goal, each step remains to be carefully optimized, and particular attention must be dedicated to the construction of the 1,4dihydropyrimidine skeleton on the support, as, in our hands, that reaction appeared to be highly sensitive to small variations in the experimental conditions.

\section{Experimental Section}

General Procedures. ${ }^{1} \mathrm{H}$ and ${ }^{13} \mathrm{C}$ NMR spectra were obtained using a Bruker AMX-300 spectrometer $\left(300 \mathrm{MHz}\right.$ for ${ }^{1} \mathrm{H}$ and $75 \mathrm{MHz}$ for ${ }^{13} \mathrm{C}$ at $\left.7 \mathrm{~T}\right)$; chemical shifts $(\delta)$ are given in ppm using TMS as internal reference. IR spectra were recorded on a Perkin-Elmer FTIR 1760K spectrometer. Melting points (not corrected) were determined on a Electrothermal 9100 apparatus. Solvents are commercially available (Aldrich Co, Acros Organics) and were used without further purification. Yields were calculated on the $\mathrm{Cl}$ content of the Merrifield's resin. Elemental analyses were performed at the Station de Haute-Belgique, Libramont-Chevigny, Belgium.

Ethyl 2-dimethylamino-4,6-diphenylpyrimidine-5-carboxylate (10). Heating under reflux for $16 \mathrm{~h}$ a mixture of Merrifield's resin ( $5 \mathrm{~g} ; 2.0 \mathrm{meq}$ of chlorine per $\mathrm{g}$ ) and thiourea (3,8 g; 50 mmol) in ethanol $\left(40 \mathrm{~mL}\right.$ ) yielded 5 (IR: $\left.3435 ; 1635 ; 1418 ; 1019 ; 754 \mathrm{~cm}^{-1}\right)$. Heating under reflux for $24 \mathrm{~h}$ a mixture of 5 , ethyl 3-aryl-2-benzoylpropenoate ${ }^{8}(6 ; 40 \mathrm{mmol})$, and sodium hydrogenocarbonate $(3.36 \mathrm{~g} ; 40 \mathrm{mmol})$ in DMF $(50 \mathrm{~mL})$ yielded $7(\mathrm{Ar}=\mathrm{Ph}$, IR: $3285 ; 1679$; $\left.1490 ; 1446 ; 1068 \mathrm{~cm}^{-1}\right)$. An aqueous solution $(40 \mathrm{~mL})$ of ceric ammonium nitrate $(21.92 \mathrm{~g} ; 40$ mmol) was slowly added to a suspension of 7 in dichloromethane $(20 \mathrm{~mL})$; stirring the mixture at $\mathrm{rt}$ for $16 \mathrm{~h}$ yielded $8\left(\mathrm{Ar}=\mathrm{Ph}\right.$, IR: 1723; 1510;1489;1211;1055 $\left.\mathrm{cm}^{-1}\right)$. Stirring at $\mathrm{rt}$ for $24 \mathrm{~h} \mathrm{a}$ mixture of 8 and $\mathrm{m}$-CPBA $(6.90 \mathrm{~g} ; 40 \mathrm{mmol})$ in dichloromethane $(50 \mathrm{~mL})$ yielded $9(\mathrm{Ar}=\mathrm{Ph}$, IR: $\left.1728 ; 1536 ; 1221 ; 1119 ; 695 \mathrm{~cm}^{-1}\right)$. A mixture of 9 and $\mathrm{N}, \mathrm{N}$-dimethylamine ( $40 \%$ in water, $6.3 \mathrm{~mL} ; 50 \mathrm{mmol})$ in ethanol $(10 \mathrm{~mL})$ was heated under reflux for $16 \mathrm{~h}$. After filtration, the solvents were removed under reduced pressure and the residue was recrystallized from a mixture of ethanol and water (3/1) to afford 10. M.p.: $172-4{ }^{\circ} \mathrm{C}$. IR (KBr): 3060;2979; $1718 \mathrm{~cm}^{-1} .{ }^{1} \mathrm{H}$ NMR ( $\left.\mathrm{CDCl}_{3}\right)$ : 0.89 (t, J = $\left.7 \mathrm{~Hz}, 3 \mathrm{H}\right) ; 3.29$ (s, $\left.6 \mathrm{H}\right) ; 3.96$ (q, J = 7 Hz; $\left.2 \mathrm{H}\right) ; 7.42$ (m, $\left.6 \mathrm{H}\right) ; 7.67$ $(\mathrm{m}, 4 \mathrm{H}) \mathrm{ppm} .{ }^{13} \mathrm{C}$ NMR $\left(\mathrm{CDCl}_{3}\right): 13.3 ; 31.0 ; 61.2 ; 114.9 ; 128.0 ; 128.3 ; 129.9 ; 139.1 ; 161.4$; $166.3 ; 169.2 \mathrm{ppm}$.

$\mathrm{C}_{21} \mathrm{H}_{21} \mathrm{~N}_{3} \mathrm{O}_{2}, 347.41$, calc. C, 72.60; H, 6.09; N, 12.10. Found C, 72.09; H, 6.11; N, 11.98. 
Ethyl 2-phenylamino-4,6-diphenylpyrimidine-5-carboxylate (11) was prepared according to the procedure described for $\mathbf{1 0}$, using aniline for the displacement.

M.p. (EtOH): $172-4{ }^{\circ} \mathrm{C}$. IR (KBr): 3398; 3055; 2980; $1718 \mathrm{~cm}^{-1} .{ }^{1} \mathrm{H}$ NMR $\left(\mathrm{CDCl}_{3}\right): 0.91(\mathrm{t}, \mathrm{J}=7$ $\mathrm{Hz}, 3 \mathrm{H}) ; 4.00$ (q, J = 7 Hz, $2 \mathrm{H}) ; 7.02(\mathrm{t}, \mathrm{J}=11 \mathrm{~Hz}, 1 \mathrm{H}) ; 7.30-7.51$ (m, $10 \mathrm{H}) ; 7.62-7.78$ (m, 4 H) ppm. ${ }^{13} \mathrm{C} \mathrm{NMR}\left(\mathrm{CDCl}_{3}\right): 13.4 ; 61.5 ; 117.3 ; 119.1 ; 128.1 ; 128.4 ; 128.8 ; 129.8 ; 138.1 ; 139.1$; $158.9 ; 166.1 ; 168.6$ ppm. $\mathrm{C}_{25} \mathrm{H}_{21} \mathrm{~N}_{3} \mathrm{O}_{2}$, 395.46, calc. C, 75.90; H, 5.35; N, 10.63. Found C, 75.57; H, 5.40; N, 10.98 .

Ethyl 2-(4-chlorophenyl)amino-4,6-diphenylpyrimidine-5-carboxylate (12) was prepared according to the procedure described for 10, using 4-chloroaniline for the displacement. M.p. (EtOH/H$\left./ \mathrm{H}_{2} \mathrm{O}-2 / 1\right): 226-8{ }^{\circ} \mathrm{C}$. IR (KBr): 3404; 3066; 2984; $1716 \mathrm{~cm}^{-1} .{ }^{1} \mathrm{H}$ NMR $\left(\mathrm{CDCl}_{3}\right)$ : $0.91(\mathrm{t}, \mathrm{J}=7 \mathrm{~Hz}, 3 \mathrm{H}) ; 3.99(\mathrm{q}, \mathrm{J}=7 \mathrm{~Hz}, 2 \mathrm{H}) ; 7.30-7.53(\mathrm{~m}, 8 \mathrm{H}) ; 7.69$ (m, $6 \mathrm{H})$ ppm. ${ }^{13} \mathrm{C}$ NMR $\left(\mathrm{CDCl}_{3}\right): 13.4 ; 61.6 ; 117.6 ; 120.4 ; 127.4 ; 128.1 ; 128.4 ; 128.7 ; 129.9 ; 137.7 ; 137.9 ; 158.7 ; 166.1$; 168.5 ppm. $\mathrm{C}_{25} \mathrm{H}_{20} \mathrm{ClN}_{3} \mathrm{O}_{2}$, 429.91, calc. C, 69.85; H, 4.69; N, 9.78. Found C, 70.09; H, 4.41; N, 10.08 .

Ethyl 2-(4-methoxyphenyl)amino-4,6-diphenylpyrimidine-5-carboxylate (13) was prepared according to the procedure described for 10, using 4-methoxyaniline for the displacement. M.p. (EtOH): $186-7^{\circ} \mathrm{C}$. IR (KBr): 3399; 3057; 2979; $1714 \mathrm{~cm}^{-1} .{ }^{1} \mathrm{H}$ NMR $\left(\mathrm{CDCl}_{3}\right): 0.90$ (t, J = 7 $\mathrm{Hz}, 3 \mathrm{H}) ; 3.80$ (s, $3 \mathrm{H}) ; 3.98$ (q, J = $7 \mathrm{~Hz}, 2 \mathrm{H}) ; 6.89$ (d, J = $11 \mathrm{~Hz}, 2 \mathrm{H}) ; 7.38-7.53$ (m, $8 \mathrm{H})$; 7.60-7.76 (m, $4 \mathrm{H})$ ppm. ${ }^{13} \mathrm{C} \mathrm{NMR}\left(\mathrm{CDCl}_{3}\right)$ : 13.4; 55.4; 61.4; 114.0; 116.9; 121.2; 128.1; 128.3; $129.7 ; 132.3 ; 138.3 ; 155.4 ; 159.2 ; 166.2 ; 168.7$ ppm. $\mathrm{C}_{26} \mathrm{H}_{23} \mathrm{~N}_{3} \mathrm{O}_{2}$, 425.49, calc. C, 73.39; $\mathrm{H}$, $5.45 ; \mathrm{N}, 9.88$. Found C, 73.09; H, 5.11; N, 9.98.

Ethyl 4,6-diphenyl-2-(pyrrolidin-1-yl)pyrimidine-5-carboxylate (14) was prepared according to the procedure described for 10, using pyrrolidine for the displacement.

M.p. $\left(\mathrm{EtOH} / \mathrm{H}_{2} \mathrm{O}-3 / 2\right): 248-50{ }^{\circ} \mathrm{C}$. IR (KBr): 3061; 2977; $1715 \mathrm{~cm}^{-1} .{ }^{1} \mathrm{H} \mathrm{NMR}\left(\mathrm{CDCl}_{3}\right): 0.89$ (t, $\mathrm{J}=7 \mathrm{~Hz}, 3 \mathrm{H}) ; 2.01(\mathrm{~m}, 4 \mathrm{H}) ; 3.71(\mathrm{~m}, 4 \mathrm{H}) ; 3.92$ (q, J = $7 \mathrm{~Hz}, 2 \mathrm{H}) ; 7.42$ (m, $6 \mathrm{H}) ; 7.65$ (m, 4 H) ppm. ${ }^{13} \mathrm{C} \mathrm{NMR}\left(\mathrm{CDCl}_{3}\right): 13.7 ; 25.7 ; 47.1 ; 61.4 ; 114.1 ; 128.3 ; 128.4 ; 129.6 ; 139.4 ; 159.6$; 166.0; 169.7 ppm. $\mathrm{C}_{23} \mathrm{H}_{23} \mathrm{~N}_{3} \mathrm{O}_{2}, 373.45$, calc. C, 73.97; H, 6.21; N, 11.25. Found C, 74.22; H, $6.18 ; \mathrm{N}, 11.57$.

Ethyl 2-(morpholin-4-yl)-4,6-diphenylpyrimidine-5-carboxylate (15) was prepared according to the procedure described for 10, using morpholine for the displacement.

M.p. $\left(\mathrm{EtOH} / \mathrm{H}_{2} \mathrm{O}-1 / 1\right): 232-4{ }^{\circ} \mathrm{C}$. IR (KBr): 3065; 2980; $1717 \mathrm{~cm}^{-1} .{ }^{1} \mathrm{H} \mathrm{NMR}\left(\mathrm{CDCl}_{3}\right): 0.94$ (t, J $=7 \mathrm{~Hz}, 3 \mathrm{H}) ; 3.77(\mathrm{~m}, 4 \mathrm{H}) ; 3.94(\mathrm{~m}, 8 \mathrm{H}) ; 7.44(\mathrm{~m}, 6 \mathrm{H}) ; 7.63(\mathrm{~m}, 4 \mathrm{H}) \mathrm{ppm} .{ }^{13} \mathrm{C} \mathrm{NMR}$ $\left(\mathrm{CDCl}_{3}\right): 13.4 ; 44.2 ; 61.3 ; 66.9 ; 114.9 ; 128.1 ; 128.2 ; 129.5 ; 138.7 ; 160.4 ; 165.8 ; 169.1$ ppm. $\mathrm{C}_{23} \mathrm{H}_{23} \mathrm{~N}_{3} \mathrm{O}_{3}$, 389.45, calc. C, 70.93; H, 5.95; N, 10.79. Found C, 70.58; H, 6.23; N, 11.05 .

Ethyl 2-butylamino-4,6-diphenylpyrimidine-5-carboxylate (16) was prepared according to the procedure described for 10, using butylamine for the displacement.

M.p. (EtOH/H$\left./ \mathrm{H}_{2} \mathrm{O}-1 / 1\right)$ : $198-9{ }^{\circ} \mathrm{C}$. IR (KBr): 3397; 3065; 2980; $1717 \mathrm{~cm}^{-1}$. ${ }^{1} \mathrm{H}$ NMR $\left(\mathrm{CDCl}_{3}\right)$ : $0.87(\mathrm{t}, \mathrm{J}=7 \mathrm{~Hz}, 3 \mathrm{H}) ; 0.96(\mathrm{t}, \mathrm{J}=7 \mathrm{~Hz}, 3 \mathrm{H}) ; 1.33-1.76(\mathrm{~m}, 4 \mathrm{H}) ; 3.53(\mathrm{t}, \mathrm{J}=7 \mathrm{~Hz}, 2 \mathrm{H}) ; 3.96$ (q, J = $7 \mathrm{~Hz}, 2 \mathrm{H}) ; 7.42(\mathrm{~m}, 6 \mathrm{H}) ; 7.62(\mathrm{~m}, 4 \mathrm{H}) \mathrm{ppm} .{ }^{13} \mathrm{C} \mathrm{NMR}\left(\mathrm{CDCl}_{3}\right)$ : 13.4; 13.8; 20.0; 31.7; 
$41.0 ; 61.2 ; 115.2 ; 128.0 ; 128.2 ; 129.5 ; 138.6 ; 161.4 ; 166.4 ; 169.0$ ppm. $\mathrm{C}_{23} \mathrm{H}_{25} \mathrm{~N}_{3} \mathrm{O}_{2}, 375.47$, calc. C, 73.58; H, 6.71; N, 11.19. Found C, 73.25; H, 6.51; N, 11.58.

Ethyl 2-dimethylamino-4-(4-nitrophenyl)-6-phenylpyrimidine-5-carboxylate (17) was prepared according to the procedure described for 10, using 4-nitrobenzaldehyde as the aldehyde component and dimethylamine for the displacement.

M.p. (EtOH): $275-7^{\circ} \mathrm{C}$. IR (KBr): 3068; 2980; 1719; 1519; $1345 \mathrm{~cm}^{-1} .{ }^{1} \mathrm{H}$ NMR $\left(\mathrm{CDCl}_{3}\right): 0.92$ (t, J = $7 \mathrm{~Hz}, 3 \mathrm{H}) ; 3.31$ (s, $6 \mathrm{H}) ; 3.94$ (q, J = $7 \mathrm{~Hz}, 2 \mathrm{H}) ; 7.47$ (m, $3 \mathrm{H}) ; 7.62$ (m, $2 \mathrm{H})$; 7.80 (d, J = $11 \mathrm{~Hz}, 2 \mathrm{H}) ; 8.24$ (d, J = $11 \mathrm{~Hz}, 2 \mathrm{H}) \mathrm{ppm} .{ }^{13} \mathrm{C} \mathrm{NMR}\left(\mathrm{CDCl}_{3}\right)$ : 13.4; 37.0; 61.4; 113.4; 123.3; $128.2 ; 128.3 ; 129.3 ; 129.6 ; 138.7 ; 145.3 ; 148.2 ; 160.9 ; 163.7 ; 166.4 ; 168.8$ ppm. $\mathrm{C}_{21} \mathrm{H}_{20} \mathrm{~N}_{4} \mathrm{O}_{4}$, 392.41, calc. C, 64.28; H, 5.14; N, 14.28. Found C, 64.12; H, 5.11; N, 14.49.

Ethyl 2-dimethylamino-4-(4-methoxyphenyl)-6-phenylpyrimidine-5-carboxylate (18) was prepared according to the procedure described for 10, using 4-methoxybenzaldehyde as the aldehyde component and dimethylamine for the displacement.

M.p. (EtOH): $158-9{ }^{\circ} \mathrm{C}$. IR (KBr): 3054; 2969; $1714 \mathrm{~cm}^{-1} .{ }^{1} \mathrm{H}$ NMR $\left(\mathrm{CDCl}_{3}\right): 0.92$ (t, J = $7 \mathrm{~Hz}, 3$ $\mathrm{H}) ; 3.31$ (s, $6 \mathrm{H}) ; 3.85$ (s, $3 \mathrm{H}) ; 3.97$ (q, J = 7 Hz, $2 \mathrm{H}) ; 6.96$ (d, J = $11 \mathrm{~Hz}, 2 \mathrm{H})$; 7.38-7.47 (m, 3 $\mathrm{H}) ; 7.60-7.69$ (m, $4 \mathrm{H}) \mathrm{ppm} .{ }^{13} \mathrm{C} \mathrm{NMR}\left(\mathrm{CDCl}_{3}\right)$ : 13.8; 37.2; 55.6; 61.4; 113.6; 113.8; 128.4; $129.5 ; 130.1 ; 131.6 ; 139.5 ; 161.0 ; 161.3 ; 165.1 ; 165.8 ; 170.1$ ppm. $\mathrm{C}_{22} \mathrm{H}_{23} \mathrm{~N}_{3} \mathrm{O}_{3}, 377.44$, calc. C, 70.01; H, 6.14; N, 12.72. Found C, 69.84; H, 6.11; N, 11.96.

\section{Acknowledgments}

One of us (N.L.) is grateful to the Belgian ministry of public health for a financial support.

\section{References}

1. (a) Vanden Eynde, J.J.; Audiart, N.; Canonne, V.; Michel, S.; Van Haverbeke, Y.; Kappe, C.O. Heterocycles 1997, 45, 1967. (b) Vanden Eynde, J.J.; Hecq, N.; Kataeva, O.; Kappe, C.O. Tetrahedron 2001, 57, 1785.

2. (a) Cho, H.; Ueda, M.; Shima, K.; Mizuno, A.; Hayashimatsu, M.; Ohnaka, Y.; Takeuchi, Y.; Hamaguchi, M.; Aisaka, K.; Hidaka, T.; Kawai, M.; Takeda, M.; Ishihara, T.; Funahashi, K.; Satoh, F.; Morita, M.; Noguchi, T. J. Med. Chem. 1989, 32, 2399. (b) Rovnyak, G.C.; Atwal, K.S.; Hedberg, A.; Kimball, S.D.; Moreland, S.; Gougoutas, J.Z.; O’Reilly, B.C.; Schwartz, J.; Malley, M.F. J. Med. Chem. 1992, 35, 1452.

3. Hurst, E.; Hull, R. J. Med. Pharm. Chem. 1961, 98, 275.

4. Kuno, A.; Sugiyama, Y.; Katsuta, K; Kamitani, T.; Takasugi, H. Chem. Pharm. Bull. 1992, 40, 1452.

5. Snider, B.B.; Shi, Z. J. Org. Chem. 1993, 58, 3828.

6. O’Reilly, B.C.; Atwal, K.S. Heterocycles 1987, 26, 1185. 
7. (a) Biginelli, P. Gazz. Chim. Ital. 1893, 23, 360. (b) Kappe, C.O. Tetrahedron 1993, 49, 6937. (c) Kappe, C.O. Eur. J. Med. Chem. 2000, 35, 1043. (d) Kappe, C.O. Acc. Chem. Res. 2000, 33, 879.

8. Milcent, R.; Malanda, J.C.; Barbier, G.; Vaissermann, J. J. Heterocycl. Chem. 1997, 34, 329.

9. Pfister, J.R. Synthesis 1990, 689.

10. Früchtel, J.S.; Jung, G. Angew. Chem. Int. Ed. Engl. 1996, 35, 17.

11. Obrecht, D.; Abrecht, C.; Grieder, A.; Villigordo, J.M. Helv. Chim. Acta 1997, 80, 65.

12. Sar, C.P.; Hankovszky, O.H.; Jerkovich, G.; Pallagi, I.; Hideg, K. ACH-Models in Chemistry 1994, 131, 363.

13. In addition to the strategy of Obrecht (reference 11), other solid-phase approaches to pyrimidine derivatives have been described by, i. a., (a) Wipf, P.; Cunningham, A. Tetrahedron Lett. 1995, 36, 7819. (b) Koloziej, S.A.; Hamper, B.C. Tetrahedron Lett. 1996, 37, 5277. (c) Studer, A.; Jeger, P.; Wipf, P.; Curran, D.P. J. Org. Chem. 1997, 62, 2917. (d) Marzinzik, A.L.M.; Felder, E.R. J. Org. Chem. 1998, 63, 723. (e) Kappe, C.O. Bioorg. Med. Chem. Lett. 2000. 10, 49. 